\title{
COLECCIÓN ALINEAMIENTOS: APROXIMACIONES A LA BIOCOMPUTACIÓN DESDE EL ARTE
}

\author{
Eloi Puig Mestres \\ Universitat de Barcelona, Departament de Pintura
}

\section{Resumen}

Este artículo presenta un proyecto artístico que trata de mostrar algunos resultados de la experiencia desarrollada gracias a la colaboración del equipo de Genómica Computacional ${ }^{1}$ dirigido por D. Torrents del BSC-CNS ${ }^{2}$ de la Universidad Politécnica de Barcelona. La Colección Alineamientos consta de una serie cuadernos que comparan textos científicos y literarios. Se trata de una visualización en torno a la relación existente entre la código gramátical de un texto y la codificación genética a través de las cadenas de ADN. Un Alineamiento de secuencias en bioinformática es una forma de representar y comparar dos o más secuencias de ADN para resaltar zonas de semejanza que indican relaciones funcionales o evolutivas entre los genes. Nuestro objetivo fue modificar este método para poder comparar texto gramatical, caracteres que siguen un conjunto de reglas y principios que gobiernan el uso de una lengua determinada.

\section{Palabras-clave: METAMÉTODO; INVESTIGACIÓN ARTÍSTICA; ALINEAMIENTO DE SECUENCIAS; ARTE Y CIENCIA; PERFORMANCE}

\section{ALIGNMENTS COLLECTION: BIOCOMPUTATION APPROACHES FROM ART}

\section{Abstract}

This paper aims to present an artistic project that show some experiences developed through collaboration Computational Genomics team led by David Torrents from BSC at the Polytechnic University of Barcelona. The Alignments Collection is a set a series notebooks comparing scientific and literary texts. This is a visualization of two artists from the University of Barcelona about the relationship between a grammatical text and DNA code. A Sequence Alignment in bioinformatics is a way to represent and compare two or more DNA sequences to highlight areas of similarity indicating functional or evolutionary relationships between genes. Our goal was to modify this method to compare, not DNA strands, but grammatical text, characters that follow rules and principles, which control the use of a particular language.

Keywords: METAMETHOD; ARTISTIC RESEARCH; SEQUENCE ALIGNMENT; ART AND SCIENCE; PERFORMANCE

\footnotetext{
Puig Mestres, Eloi. 2016. "Colección Alineamientos: Aproximaciones a la biocomputación desde el Arte”. AusArt 4(1): pp-pp. 187-196 DOI: 10.1387 /ausart.16700
}

\section{AUSART}


"Todo el arte de Kafka consiste en obligar al lector a releer. Sus desenlaces, o la ausencia de desenlaces, sugieren explicaciones, pero que no se revelan claramente y que exigen, para que parezcan fundadas, una nueva lectura del relato desde otro ángulo. $A$ veces hay una doble posibilidad de interpretación, de donde surge la necesidad de dos lecturas. Eso es lo que buscaba el autor. Pero sería un error querer interpretar todo detalladamente en Kafka."

(Camus [1942] 2002, 135)

\section{INTRODUCCIÓN}

Un subgrupo del equipo de investigación artística de la Facultad de Bellas Artes de la Universidad de Barcelona han estado trabajando con el equipo de Genómica Computacional dirigido por David Torrents. El marco del proyecto de investigación Metamétodos ${ }^{3}$, nuestro equipo se centra en nuevas estrategias metodológicas de visualización e interpretación de los procesos del arte. Busca conectar con las estructuras de investigación que otras disciplinas aplican en sus proyectos, para de esta manera, poder entenderlas, leerlas y posteriormente, en una segunda etapa, poder adaptarlas y modificarlas ya desde una perspectiva creativa, permitiendo que surja un pensamiento original, nuevas ideas y nuevos conceptos. Se trata, en definitiva, de un metamétodo que trata (desde el arte como proceso y producción) encontrar nuevas relaciones entre la teoría y la práctica, entre la ciencia y la tecnología creando una serie de enlaces multidisciplinares basados en la investigación, estableciendo vínculos de similitud y diferencia entre el método científico y el artístico.

La relación con el equipo de Genómica Computacional se inició en septiembre del año 2011 y se realizaron 7 diferentes encuentros hasta junio de 2012. El objetivo principal fue conocer qué tipo de investigaciones se estaban desarrollando y qué métodos utilizaban. Construimos un blog 4 donde íbamos transcribiendo cada uno de nuestros encuentros y poner de relieve la importancia del proceso para llegar a detectar el método que utilizaríamos para dar respuesta a lo que nosotros, como artistas veíamos y entendíamos. 
El diálogo fue muy fructuoso ya que, a partir de entonces, entendimos la relevancia del Alineamiento de Secuencias como principio del trabajo desarrollado por el grupo de investigadores del equipo de Genómica. Este método de alineamiento fue modificado para poder comparar, no cadenas de $A D N$, sino texto gramatical, aquellos conjuntos de caracteres que siguen un conjunto de reglas y principios que gobiernan el uso de una lengua concreta determinada.

De esta manera una vez verificado y acordado una línea posible de convergencia entre los investigadores de genómica y nosotros como intérpretes del ámbito artístico, iniciamos una serie de experimentos basados en el alineamiento, que dieron lugar un conjunto muy variado de propuestas. Todas ellas han ido siendo mostradas en diferentes espacios expositivos y bajo múltiples formatos, como por

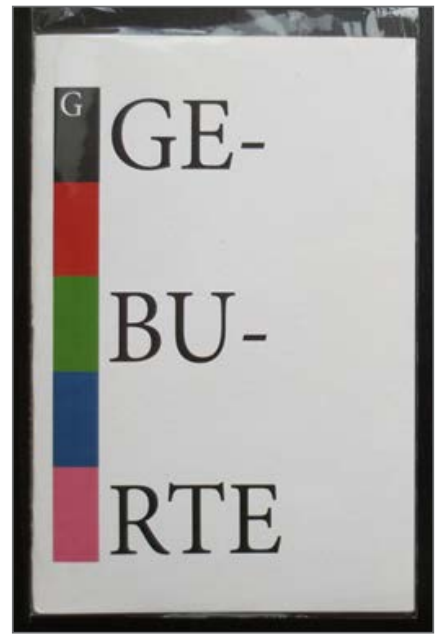

Fig.1: Portada de Geburte. Primera publicación realizada mediante el método de Alineamiento de secuencias. Año 2012. ejemplo:

1. Publicación Geburte. Alineación de las respuestas de los diferentes miembros del equipo de genómica a nuestras preguntas durante el proceso de conocer su particular actividad, así como también algunas preguntas específicas al investigador principal respecto al propio funcionamiento del grupo. Marzo 2012. Proyecto Geburte mostrado en la Feria Art Libris (Barcelona)

2. Sara sings. Alineación entre la letra de una canción infantil y la transcripción del canto de la misma canción hecha por una niña de 3 años. Mostrado en el marco de una exposición colectiva basada en proyectos artísticos entorno al error. Sala Corretger 5. Barcelona. Noviembre 2012.

3. TE LA (Text Local Alignment). Alineación entre la transcripción de la locución de dos vídeos domésticos que hablan sobre la relación entre ciudad y ciencia. Arts Santa Mónica. Barcelona. Noviembre. 2012.

4. Renga-Ronda. Alineación entre el poema de un poeta escocés que habla sobre el paisaje de los Highlands del norte de Escocia y el 
poema de un poeta catalán que habla sobre la Barcelona nocturna. Publicado como libro en formato DinA3. HICA Highland Institute for Contemporary Art, Escocia. Abril 2012.

5. The White Wall. Lectura de una frase de Jorge Guillén de su libro El argumento de la obra, sobre el espacio de lectura en el que se encuentra el texto escrito. Enero 2013. Galeria Ferran Cano.

6. Wagensber's Alphabet. Alineación de aforismos de Jorge Wagensber. Fresados planográficos sobre planchas blancas de PVC. Julio 2014.

7. HM. Interpretación de un fragmento del cuaderno HM a través del software roloc (traducción de imágenes en franjas cromáticas). Sala d'exposicions Centre Cultural El Carme. Setiembre. 2014.

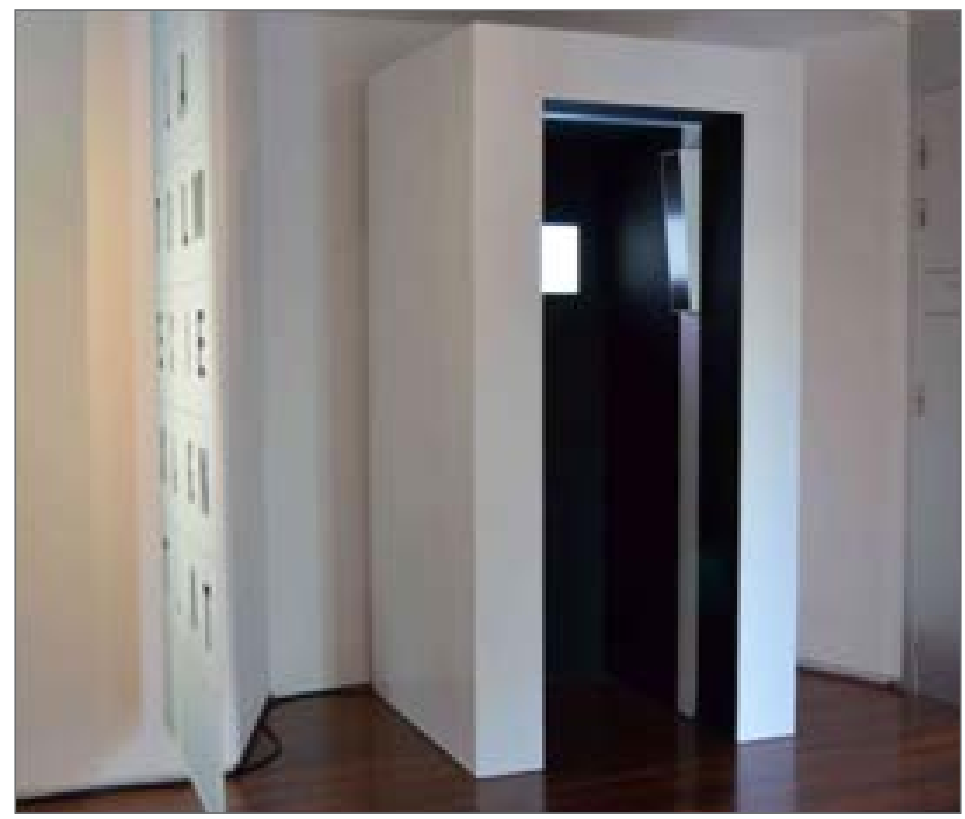

Fig. 2: Instalación de la pieza TE LA (Text Local Alignment), en el marco de la exposición colectiva Science of the City. Centre d'Art Santa Mònica. Barcelons. 212. 


\section{UN PARTICULAR USO DEL MÉTODO DE ALINEAMIENTO DE SECUENCIAS}

El ser humano se origina con una sola célula formada a partir de un óvulo y un espermatozoide, pero para que esta célula única se convierta en los miles de millones de células que forman el cuerpo humano, y toda esta máquina celular funcione de una forma coordinada, hay un código con instrucciones, el ADN. El código o texto del ADN, el genoma, a diferencia de nuestro alfabeto no está escrito con 28 letras sino con cuatro, sin signos de puntuación, con espacios en blanco. Pero la investigación en bioinformática hace necesarias un número de letras igual al número de letras del alfabeto, porque su campo de estudio requiere más variaciones. Es de esta manera como ambos códigos, el genético y el gramático, utilizan 28 caracteres.

Con el objetivo de detectar cambios genéticos entre cadenas de ADN, el Alineamiento de Secuencias es el procedimiento básico de análisis por comparación. Es básico pero no sencillo, ya que este método trata de dar solución a los exigentes cálculos que se derivan de los estudios genéticos. Existe una gran variedad de software que ofrece diferentes formas de alineamiento, se diferencian en dos grandes grupos, los alineamientos locales o los globales. Por lo general, un alineamiento selecciona la parte más coincidente entre ambas líneas de código, marca en mayúsculas las coincidencias totales y las coincidencias aproximadas en minúsculas. Las probabilidades de coincidencias son regidas por una matriz que, al mismo tiempo, contiene una programación específica y determinada por el objetivo mismo de los intereses de la investigación.

Los alineamientos se representan normalmente con un formato gráfico y de texto. En casi todas las representaciones de alineamientos, las secuencias se escriben en filas de forma que los residuos alineados aparecen en columnas sucesivas. En los formatos de texto, las columnas alineadas contienen caracteres idénticos o similares, estos últimos indicados con sistema de símbolos de conservados... Muchos programas de visualización de secuencias utilizan también esquemas coloreados para mostrar información de las propiedades de los elementos secuencia individuales ${ }^{5}$.

Utilizar el Alineamiento de secuencias supuso establecer el punto de partida de nuestra investigación. Los códigos de la bioinformática y la gramática de los textos utilizan el mismo número de caracteres para poder determinar diferencias con una precisión y exactitud científica. Esa coincidencia la aprove- 
chamos para sustituir los 28 caracteres del código genético por los 28 caracteres de nuestro alfabeto. Utilizando el mismo software bioinformático alineamos texto gramático, literario. Dicho software, llamado Swat5, fue facilitado por el investigador genómico Santiago González (miembro del BSC). Éste mismo nos introdujo en su uso, limitando y adaptando las miles maneras de ser aplicado según las pautas previamente consensuadas. Los resultados de estas alineaciones serán mostradas con el mismo aspecto gráfico y formal con el que los científicos realizan sus estudios, estadísticas y conclusiones. Veremos pues como también, en nuestras alineaciones, aparecerán en mayúsculas las coincidencias totales y las coincidencias aproximadas en minúsculas, espacios en blanco y guiones negros para ajustarlas. Serán alineamientos de dos textos teniendo en cuenta que las proteínas de las cadenas de ADN se representan utilizando un código basado en 28 caracteres de texto genético, nuestro procedimiento sustituye estas cadenas de proteínas de ADN por texto gramático.

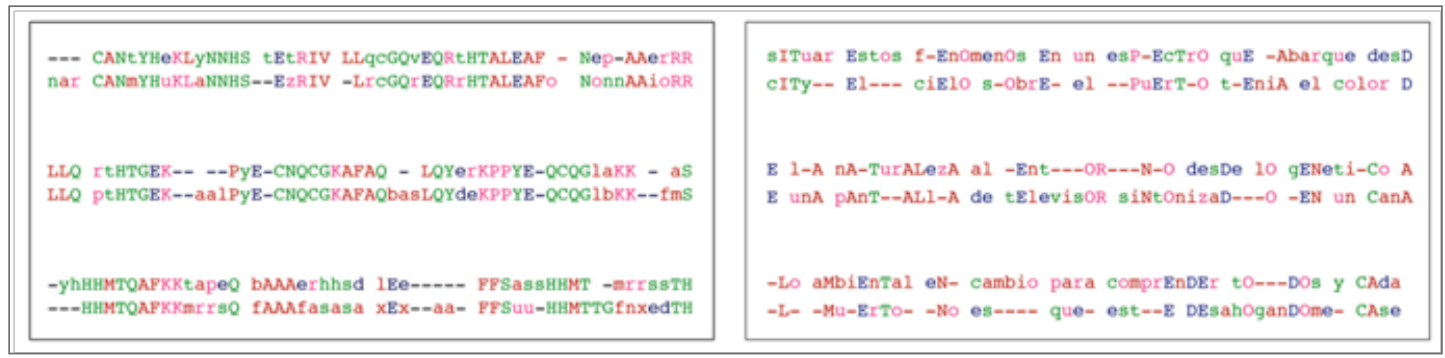

Fig.3: Ejemplos de alineaciones a través del mismo software Swat5 (cedido e instalado por los bioinformáticos en nuestros ordenadores). Imagen de la izquierda: Alineación de códigos de ADN. Imagen de la derecha: Alineación de textos gramaticales.

\section{COLECCIÓN ALINEAMIENTOS}

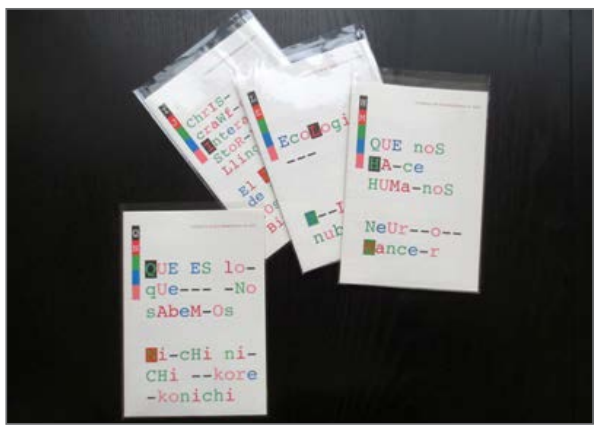

Fig. 4: Los cuatros números de la Colección Alineamientos. Años 2014 y 2015.
La Colección Alineamientos se presenta en forma de cuaderno y consta, hasta el momento, de 4 números autoeditados. Cada publicación está formada por la alineación de un fragmento de dos libros, cada uno de ellos es seleccionado por un científico y por alguien del ámbito de las humanidades, conformando parejas que muestran sus propios e individuales intereses. 
Este proyecto quiere evaluar la relación entre las dos culturas, la ciencia y las humanidades, utilizando el método del Alineamiento de Secuencias para visualizar aproximaciones al concepto de Tercera Cultura del John Brockman ${ }^{6}$. La Tercera Cultura, según el editor John Brockman, es la superación de las dos culturas: la científica y la humanística, para integrarlas en una tercera en la que sólo hay conocimiento.

El procedimiento para llevar a cabo el alineamiento sigue siempre una misma pauta. En primer lugar para cada número, nosotros, los autores, hemos conectado con dos agentes diferentes, uno relacionado profesionalmente con el ámbito científico y otro con el ámbito humanístico y/o artístico. A cada uno de ellos se les ha preguntado por una de las lecturas que han considerado importantes o que han detectado una especial afinidad y, posteriormente, nos han comunicado su referencia bibliográfica. Una vez que obtenemos el texto o libro referenciado por esos dos agentes, hemos seleccionado un fragmento de cada uno para posteriormente procesarlos mediante el Swat5.

Los resultados de estos alineamientos han sido formalizados mediante una publicación sobre papel DinA3 a dos caras, en color y con dos pliegues que lo convierten en una cuaderno DinA5. Hasta el momento tenemos los siguientes 4 números:

no1 --- QN

¿Qué es lo que no sabemos? de llya Prigogine y Nichi nichi kore konichi de John Cage seleccionado por Aleix Molet y Eloi Puig respectivamente.

no2 --- HM

Que es lo que nos hace humanos de Mad Ridely y Neuromancer de William Gibson seleccionados por David Torrents y David Casacuberta respectivamente.

no3 --- LE

Ecología de Ramón Margalef y El Danubio de Claudio Magris seleccionado por Santiago González y Vitor Magalhaes respectivamente.

no4 --- IJ

Chris Crawford On Interactive Storytelling de Chris Crawford + El jardín de senderos que se bifurcan de Jorge Luis Borges seleccionado por Arturo Galbete y Ignasi Pàmies respectivamente. 


\section{CONCLUSIONES}

Como aquí se ha expuesto, y quizás demostrado, sólo una investigación que interfecunde, es decir, que englobe ciencias y humanidades, puede superar el carácter incompleto de cualquier descripción conceptual del mundo. Para poder visualizar este concepto tan complejo, hemos creado varias formalizaciones que interrelacionan a un nivel metalingüístico las estructuras del lenguaje de ciencia y ciencia ficción. Estas formalizaciones se ha realizado a partir de dos niveles de aproximación: uno pertinente al significado y otro formal.

- Aproximación de primer nivel: relaciones lingüísticas, significado. El texto resultante de la Alineación genera un nuevo texto, que contiene la misma cantidad de letras pero con un nuevo orden, que produce una lectura fragmentada. La fragmentación es la que da sentido al proyecto, porque hace que el conocimiento de los datos iniciales sea imprescindible para evaluar su significado posterior. En el caso de que no se conozcan todos los datos iniciales se deben aceptar como verdaderos. Ni las ciencias ni las humanidades conocen todos los datos iniciales para llevar a cabo su heurística.

El poeta Stéphane Mallarmé ya intuyó la importancia de no conocer todos los datos iniciales, por eso jugaba con el espacio en blanco entre palabras y letras en su célebre poema Un coup de dés, jamais n'abolirá le hasard. Nadie sabe cuál es la duración de estos espacios blancos, ni el propio Mallarmé conoce todos los datos iniciales de su poema. Mallarmé introduce el espacio en blanco entre tipografías para crear un espacio objetivo donde cada lector determina la duración y relación con la tipografía siguiente. En nuestro proyecto la heurística no se basa en la intuición, como proponía Mallarmé, sino en la matemática.

- Aproximación de segundo nivel: relaciones formales, visuales. Obtenemos un cuaderno, que forma parte de una colección que hemos denominado, de manera obvia, Colección Alineamientos. HN es el título del cuaderno y hace referencia a dos letras de los títulos de los textos seleccionados por los investigadores externos.

Es un cuaderno donde las letras reciben un color análogo al de los códigos metodológicos utilizados en genómica. Son los colores que se obtienen por fluorescencia a través de las máquinas de Secuenciación Automáticas. Cada letra de las bases $A, C, G, T$ se une a un marcador fluorescente y, al añadirse 
esta letra en la secuencia o frase, el marcador se libera y emite un haz de luz de su color; una cámara especial puede ir detectando estas chispas de luz a medida que se va formando la frase y de esta manera se obtiene la secuencia .

\section{Referencias}

Brockman, John, ed. (1995) 2000. La tercera cultura: Más allá de la revolución científica. Traducción de Ambrosio García. Barcelona: Tusquets

Camus, Albert. (1942) 2002. El mito de Sísifo : Ensayo sobre el absurdo. Traducción de Luis Echávarri. Buenos Aires: Losada

Capra, Fritjof. (1996) 2009. La trama de la vida: Una nueva perspectiva de los sistemas vivos. Traducción de David Sempau. Barcelona: Anagrama

Hayles, N. Katherine. (1991) 2000. La evolución del Caos. Traducción, Ofelia Castillo. BarceIona: Gedisa

Hofstadter, Douglas R. (1979) 2007. Gödel, Escher, Bach: Un eterno y grácil bucle. Traducción, Mario A. Usabiaga y Alejandro López Rousseau. Barcelona: Tusquets

Montfort, Nick \& Noah Wardrip-Fruin, eds. 2003. The new media reader. Cambridge MA: MIT

Planagumà, Pere et al. 2011. Matèria: Noves fronteres de la ciencia, l'art i el pensament. Encontres internacionals $\left(20^{\circ}\right.$, Barcelona, 2010). Barcelona: Arts Santa Mónica

Sarmiento, José Antonio. 2012. Música y acción: Exposición-concierto de 40 piezas para instrumentos varios: Centro José Guerrero, Granada, del 19 de octubre de 2012 al 13 de enero de 2013. Granada: Centro José Guerrero

Wagensberg Lubinski, Jorge. 1985. Ideas sobre la complejidad del mundo. Barcelona: Tusquets

Wilson, Stephen. 2003. Information arts: Intersections of Art, Science, and Technology. Cambridge MA: MIT

Notas

${ }^{1}$ http://www.bsc.es/life-sciences/computational-genomics [acceso 02.06.2015]

2 El Barcelona Supercomputing Center-Centro Nacional de Supercomputación es el centro pionero de la supercomputación en España. Su naturaleza es doble: por una parte es un centro de investigación formado por más de trescientos científicos, y por otra es un centro de servicios de supercomputación para toda la comunidad científica. También gestiona la Red Española de Supercomputación (RES). www.bsc.es

3 "Metamétodo: Metodologías compartidas y procesos artísticos en la sociedad del conocimiento HAR2010-18453 (subprograma ARTE)". Plan Nacional de I+D+i 2008-2011. Subprograma de proyectos de investigación Fundamental no orientada. Subvencionado por el Ministerio de Economía y Competitividad y la Unión Europea.

${ }^{4}$ https://geburte.wordpress.com/ [acceso 02.06.2015] 
${ }^{5}$ http://es.wikipedia.org/wiki/Alineamiento de secuencias [acceso 14.05.2015]

${ }^{6}$ John Brockman es fundador y presidente de una agencia literaria neoyorquina especializada en la promoción y gestión de derechos de autores científicos. Agente cultural de amplia trayectoria en el campo del arte, la ciencia, los libros, el software e Internet. Editor de Edge, página web donde los pensadores más destacados, líderes de lo que él llama Tercera Cultura, analizan la ciencia más vanguardista.

(Artículo recibido 10.04.16; aceptado 19.05.16) 